\title{
History of Adverse Pregnancy on Subsequent Maternal-Fetal Outcomes in Patients with Immunoglobulin A Nephropathy: A Retrospective Cohort Study from a Chinese Single Center
}

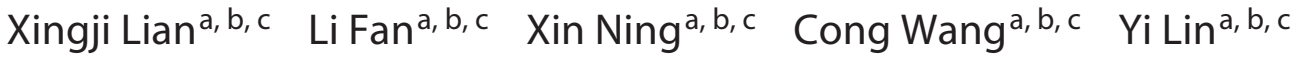 \\ Wenfang Chen ${ }^{d}$ Wei Chen ${ }^{a, b}, c$ Xueqing $\mathrm{Yu}^{\mathrm{a}}{ }^{\mathrm{a}}, \mathrm{b}, \mathrm{c}$ \\ ${ }^{a}$ Department of Nephrology, The First Affiliated Hospital, Sun Yat-sen University, Guangzhou, China; ${ }^{b} \mathrm{NHC}$ Key \\ Laboratory of Nephrology (Sun Yat-sen University), Guangzhou, China; ' Guangdong Provincial Key Laboratory of \\ Nephrology, Guangzhou, China; ${ }^{d}$ Department of Pathology, The First Affiliated Hospital, Sun Yat-sen University, \\ Guangzhou, China
}

\section{Keywords}

Immunoglobulin A nephropathy · Chronic kidney disease .

Pregnancy outcome $\cdot$ Kidney disease outcome

\begin{abstract}
Background: Gestation complications have a recurrence risk and could predispose to each other in the next pregnancy. We aimed to evaluate the relationship between a history of adverse pregnancy and maternal-fetal outcomes in subsequent pregnancy in patients with Immunoglobulin A nephropathy (IgAN). Methods: A retrospective cohort study from a Chinese single center was conducted. Pregnant women with biopsy-proven primary IgAN and aged $\geq 18$ years were enrolled and divided into the 2 groups by a history of adverse pregnancy. The primary outcome was adverse pregnancy outcome, which included maternal-fetal outcomes. Logistical regression model was used to evaluate the association of a history of adverse pregnancy with subsequent adverse maternal and fetal outcomes. Results: Ninety-one women with 100 pregnancies were included, of which 54
\end{abstract}

karger@karger.com www.karger.com/kdd

Karger ${ }^{\prime \prime} \div$
(C) 2021 The Author(s)

Published by S. Karger AG, Basel

This is an Open Access article licensed under the Creative Common Attribution-NonCommercial-4.0 International License (CC BY-NC) (http://www.karger.com/Services/OpenAccessLicense), applicable to the online version of the article only. Usage and distribution for commercial purposes requires written permission.
(54\%) pregnancies had a history of adverse pregnancy. IgAN patients with adverse pregnancy history had more composite maternal outcomes $(70.4 \%$ vs. $45.7 \%, p=0.012)$, while there was no difference in the composite adverse fetal outcomes between the 2 groups ( $55.6 \%$ vs. $45.7 \%)$ ). IgAN patients with a history of adverse pregnancy were associated with an increased risk of subsequent adverse maternal outcomes (adjusted odds ratio [OR], 2.64; 95\% Cl, 1.07-6.47). Similar results were shown in those with baseline serum albumin $<3.5 \mathrm{~g} / \mathrm{dL}, 24 \mathrm{~h}$ proteinuria $\geq 1 \mathrm{~g} /$ day, and a history of hypertension. There was no association between a history of adverse pregnancy and subsequent adverse fetal outcomes in IgAN patients (adjusted OR, 1.56; $95 \% \mathrm{Cl}, 0.63-3.87$ ). Conclusion: A history of adverse pregnancy was associated with an increased risk of subsequent adverse maternal outcomes, but not for adverse fetal outcomes in IgAN patients.

(C) 2021 The Author(s)

Published by S. Karger AG, Basel

Xingji Lian and Li Fan contributed equally to this work.
Correspondence to:

Wei Chen, chenwei99@mail.sysu.edu.cn 


\section{Introduction}

Immunoglobulin A nephropathy (IgAN) is the most common primary glomerular nephropathy worldwide, especially in Asian populations [1]. Overall, 30-40\% of patients may progress to end-stage kidney disease in 2030 years after diagnosis [2]. Most female patients with IgAN are diagnosed during the childbearing period due to its higher incidence in those aged 20-30 years [3,4]. Therefore, the impact of IgAN on mother or newborn has been a major concern.

Previous studies have showed that gestation complications, including stillbirth, spontaneous preterm birth, intrauterine growth restriction, and preeclampsia in general population have a recurrence risk and could predispose to each other in the next pregnancy $[5,6]$. It is essential for health providers to be conscious of and identify the at-risk pregnancies with a history of adverse birth outcomes, which may be helpful for antenatal surveillance and target interventions to prevent adverse outcomes in continuing pregnancy. Currently, most of the studies focused on the effects of pregnancy on kidney disease progression and risk factors associated with adverse pregnancy outcomes in patients with IgAN [7-9]. There is limited evidence regarding on the impact of a history of adverse birth outcomes on maternal-fetal outcomes and long-term renal outcome in IgAN patients. Therefore, in this study, we aimed to evaluate the association of a history of adverse pregnancy with recurrent maternal-fetal outcomes in subsequent pregnancy in IgAN patients. We also compared kidney function and proteinuria after delivery and during follow-up between IgAN patients with and without a history of adverse pregnancy outcomes.

\section{Materials and Methods}

\section{Study Design and Population}

This is an observational cohort study. Patients in the IgAN registry of the First Affiliated Hospital, Sun Yat-sen University (FAHSYSU) (http://igan.medidata.cn) during January 1, 2003 to December 31, 2019 were screened. Female pregnant patients with biopsy-proven primary IgAN and aged $\geq 18$ years were enrolled. We excluded patients who experienced pregnancy before diagnosis of IgAN, and had missing baseline or gestational information. Eligible patients were divided into 2 groups based on a history of adverse pregnancy. A history of adverse pregnancy was defined as fetal loss, pre-eclampsia, and premature birth.

Demographic and Clinical Data

Baseline demographic, clinical, and biochemistry data of the participants were collected at the time of kidney biopsy from medical charts. Demographic and clinical characteristics (age, body mass index, gestation history, and blood pressure) and biochemistry variables (hemoglobin, serum creatinine, albumin, blood urine nitrogen, and proteinuria) were collected. We also collected the medicine history of angiotensin receptor blockers, angiotensin converting enzyme inhibitors, corticosteroids, and immunosuppressant agents at the kidney biopsy. Maternal and fetal outcomes were ascertained through reviewing medical electronic health record database. Estimated glomerular filtration rate (eGFR) was calculated using Chronic Kidney Disease Epidemiology Collaboration creatinine formula [10] and renal histologic lesions were evaluated and graded according to the Oxford MEST-C classification criteria of IgA nephropathy (M, mesangial hypercellularity; E, endocapillary proliferation; S, segmental glomerulosclerosis/adhesion; T, tubular atrophy/interstitial fibrosis; and C, crescent formations) [11].

\section{Study Outcomes}

The primary outcomes were adverse pregnancy outcomes, which included adverse maternal outcomes and fetal outcomes. Adverse maternal outcomes were defined as delivery by cesarean section, premature rupture of fetal membrane, or pre-eclampsia. Adverse fetal outcomes were defined as low birth weight, preterm deliveries, fetal loss, or fetal distress.

Pre-eclampsia was diagnosed when a new-onset hypertension (blood pressure $>140 / 90 \mathrm{~mm} \mathrm{Hg}$ ) and proteinuria (24-h urine protein excretion $>0.3 \mathrm{~g}$ ) occurred after week 20 of gestation [12]. Preterm birth was defined as a live-born infant delivery with a gestational age $<37$ weeks and low birth weight was defined as an infant weight $<2.5 \mathrm{~kg}$ [13]. Fetal loss was defined as spontaneous abortion (intrauterine fetal demise before the 20th week of gestation caused by natural factors), stillbirth (intrauterine fetal demise after 20th week of gestation), neonatal death (live infant dying within 28 days after delivery), and therapeutic and induced abortions because of uncontrolled diseases [8]. We also investigated the change of maternal kidney function and proteinuria after delivery.

\section{Statistical Analysis}

Data were presented as mean \pm standard deviation (SD) or median (25th, 75th) for continuous variables, as appropriate, as well as the number (percent) or frequency for categorical variables. $\mathrm{Pa}$ tients were divided by previous history of adverse pregnancy. Baseline demographic and laboratory variables were compared between patients with and without history of adverse pregnancy using Student's $t$ test or Mann-Whitney $U$ test for continuous variables, and $\chi^{2}$ test or Fisher's exact test for categorical variables, as appropriate.

Univariate and multivariate logistic regression model was used to evaluate the association of a history of adverse pregnancy with subsequent adverse maternal and fetal outcomes. The following potential confounding covariates were adjusted in the multivariate model: age, mean arterial pressure, proteinuria, serum albumin, and eGFR.

A priori subgroup analyses were conducted and defined by baseline serum albumin $(\geq 3.5 \mathrm{~g} / \mathrm{dL}$ vs. $<3.5 \mathrm{~g} / \mathrm{dL}), 24$-h urine protein excretion ( $\geq 1 \mathrm{~g} /$ day vs. $<1 \mathrm{~g} /$ day $)$, eGFR $\left(\geq 60 \mathrm{~mL} / \mathrm{min} / 1.73 \mathrm{~m}^{2}\right.$ vs. $<60 \mathrm{~mL} / \mathrm{min} / 1.73 \mathrm{~m}^{2}$ ), and hypertension status (yes vs. no). We further compared the proteinuria and eGFR levels between patients with and without previous history of adverse pregnancy at the time of delivery and follow-up until July 1, 2020 using Whitney U test. 
Table 1. Baseline clinical characteristics at the time of kidney biopsy

\begin{tabular}{|c|c|c|c|c|}
\hline Variables & Overall & $\begin{array}{l}\text { With adverse } \\
\text { pregnancy history }\end{array}$ & $\begin{array}{l}\text { Without adverse } \\
\text { pregnancy history }\end{array}$ & $p$ value \\
\hline$N$ & 100 & 54 & 46 & \\
\hline Body mass index, $\mathrm{kg} / \mathrm{m}^{2}$ & $20.1(18.8,22.1)$ & $20.7(19.5,22.5)$ & $19.5(18.4,21.2)$ & $<0.01$ \\
\hline Time from biopsy to pregnancy, yr & $4.0(2.0,6.0)$ & $4.0(2.0,7.0)$ & $2.0(1.0,4.0)$ & $<0.01$ \\
\hline Time from biopsy to delivery, $\mathrm{yr}$ & $3.0(1.0,5.0)$ & $5.0(3.0,8.0)$ & $3.0(1.0,5.0)$ & $<0.01$ \\
\hline Systolic BP, mm Hg & $119.1(18.3)$ & $119.7(18.0)$ & $118.5(18.9)$ & 0.75 \\
\hline Diastolic BP, mm Hg & $77.0(13.7)$ & $77.0(13.1)$ & $77.0(14.5)$ & 0.99 \\
\hline Mean arterial pressure, $\mathrm{mm} \mathrm{Hg}$ & $86.7(80.0,97.0)$ & $86.7(81.3,97.0)$ & $86.8(79.7,97.0)$ & 0.54 \\
\hline \multicolumn{5}{|l|}{ Proteinuria category, $n(\%), \mathrm{g} / \mathrm{d}$} \\
\hline$<1$ & $65(65.0)$ & $35(64.8)$ & $30(65.2)$ & 0.55 \\
\hline $1-2$ & $27(27.0)$ & $16(29.6)$ & $11(23.9)$ & \\
\hline Serum albumin, $\mathrm{g} / \mathrm{dL}$ & $3.8(3.6,4.1)$ & $3.9(3.7,4.1)$ & $3.7(3.5,4.1)$ & 0.08 \\
\hline \multicolumn{5}{|l|}{ Oxford classification, ${ }^{a} n(\%)$} \\
\hline M1 & $88(100.0)$ & $47(100.0)$ & $41(100.0)$ & \\
\hline E1 & $14(15.9)$ & $7(14.9)$ & $7(17.1)$ & 0.74 \\
\hline S1 & $47(53.4)$ & $22(46.8)$ & $25(61.0)$ & 0.08 \\
\hline $\mathrm{T} 1 / 2$ & $22(25.0)$ & $10(21.3)$ & $12(29.3)$ & 0.34 \\
\hline $\mathrm{C} 1 / 2$ & $29(32.9)$ & $15(31.9)$ & $13(31.7)$ & 0.82 \\
\hline \multicolumn{5}{|l|}{ Treatment before pregnancy, $n(\%)$} \\
\hline RAS blocker treatment alone & $71(71.0)$ & $37(68.5)$ & $34(73.9)$ & 0.55 \\
\hline Glucocorticoids and/or immunosuppressive drugs & $43(43.0)$ & $23(42.6)$ & $20(43.5)$ & 0.76 \\
\hline
\end{tabular}

Data are expressed as mean and standard deviation and median and range. $p<0.05$. eGFR, estimated glomerular filtration rate; BP, blood pressure; RAS, renin-angiotensin system. ${ }^{a}$ Analysis was based on 41 patients without adverse pregnancy history and 47 patients with adverse pregnancy history.

All analyses were performed using Stata version 15 (Stata Corp LP, College Station, TX, USA) and R (version 3.6.3; Free Software Foundation Inc, www.r-project.org). A $p$ value $<0.05$ was considered as statistically significant.

\section{Results}

Clinical Characteristics of Pregnant Patients with IgAN at Baseline and the Time of Delivery

A total of 91 women with 100 pregnancies who experienced pregnancy after IgAN diagnosis were included in this study (online suppl. Fig. 1; for all online suppl. material, see www.karger.com/doi/10.1159/000520586). At the time of biopsy, compared to patients without a history of adverse pregnancy, patients with a history of adverse pregnancy had higher body mass index, and longer time from biopsy to pregnancy and delivery. However, most of base- line characteristics were similar between the 2 groups, including age, baseline serum creatinine, eGFR, proteinuria, hemoglobin, serum albumin, blood pressure, pathologic characteristics, and treatment (Table 1). At the time of delivery, patients with a history of adverse pregnancy had a higher body mass index and blood pressure, and there were not statistical differences in other characteristics between the 2 groups (online suppl. Table 1).

\section{Adverse Pregnancy Outcomes}

There were $51(51.0 \%)$ adverse fetal outcomes and 59 (59.0\%) maternal outcomes in 100 pregnancies. For fetal outcomes, patients with a history of adverse pregnancy had higher frequency of preterm birth than those without adverse pregnancy history ( $31.5 \%$ vs. $13.0 \%, p=0.04)$. However, there were no statistical differences in frequency of fetal death, low birth weight, fetal distress, and composite adverse fetal outcomes between the 2 groups. For maternal 
Table 2. Pregnancy outcomes of pregnant patients with IgA nephropathy

\begin{tabular}{|c|c|c|c|}
\hline Variables & $\begin{array}{l}\text { With adverse } \\
\text { pregnancy history }\end{array}$ & $\begin{array}{l}\text { Without adverse } \\
\text { pregnancy history }\end{array}$ & $p$ value \\
\hline \multicolumn{4}{|l|}{ Fetal outcomes, $n(\%)$} \\
\hline Fetal death & $5(9.3)$ & 9 (19.6) & 0.27 \\
\hline Preterm delivery & $17(31.5)$ & $6(13.0)$ & 0.04 \\
\hline Low birth weight & $10(18.5)$ & $7(15.2)$ & 0.73 \\
\hline Fetal distress & $4(7.4)$ & $3(6.5)$ & 0.91 \\
\hline Composite measure ${ }^{a}$ & $30(55.6)$ & $21(45.7)$ & 0.32 \\
\hline \multicolumn{4}{|l|}{ Maternal outcomes, $n(\%)$} \\
\hline Premature rupture of membranes & $8(14.8)$ & $1(2.2)$ & 0.030 \\
\hline Pre-eclampsia & $8(14.8)$ & $1(2.2)$ & 0.030 \\
\hline Delivery by cesarean section & $33(61.1)$ & $19(41.3)$ & 0.06 \\
\hline Composite measure ${ }^{a}$ & $38(70.4)$ & $21(45.7)$ & 0.012 \\
\hline
\end{tabular}

${ }^{a}$ Adverse pregnancy outcome occurred for a single pregnancy was defined as a composite measure. $p<0.05$.

Table 3. Association of a history of adverse pregnancy with fetal and maternal outcomes

\begin{tabular}{|c|c|c|c|c|c|c|c|c|}
\hline Variables & \multicolumn{4}{|c|}{ Fetal outcomes } & \multicolumn{4}{|c|}{ Maternal outcomes } \\
\hline A history of adverse pregnancy ${ }^{a}$ & 1.56 & 0.63 & 3.87 & 0.33 & 2.64 & 1.07 & 6.47 & 0.034 \\
\hline Age (per 1 year) & 0.99 & 0.88 & 1.11 & 0.83 & 0.98 & 0.88 & 1.10 & 0.74 \\
\hline Serum albumin (per $1 \mathrm{~g} / \mathrm{dL}$ ) & 0.92 & 0.82 & 1.03 & 0.17 & 0.99 & 0.89 & 1.10 & 0.82 \\
\hline eGFR (per $1 \mathrm{~mL} / \mathrm{min} / 1.73 \mathrm{~m}^{2}$ ) & 0.98 & 0.96 & 1.00 & 0.07 & 1.00 & 0.99 & 1.02 & 0.66 \\
\hline
\end{tabular}

eGFR, estimated glomerular filtration rate. ${ }^{\text {a }}$ Analysis was based on adjusted model.

outcomes, patients with a history of adverse pregnancy had higher frequency of premature rupture of membranes $(14.8 \%$ vs. $2.2 \%, p=0.03)$, pre-eclampsia ( $14.8 \%$ vs. $2.2 \%, p$ $=0.03)$, and composite adverse maternal outcomes $(70.4 \%$ vs. $45.7 \%, p=0.012$ ) than those without adverse pregnancy history, but no statistical differences were found on the frequency of delivery by cesarean section (Table 2).

\section{Effect of Pregnancy History on Subsequent Adverse Pregnancy Outcomes}

There were no associations between a history of adverse pregnancy and subsequent composite adverse fetal outcomes either in unadjusted model (OR, 1.49; 95\% CI, $0.68-3.28$ ) or adjusted model (OR, 1.56; 95\% CI, 0.633.87). For composite adverse maternal outcomes, patients with a history of adverse pregnancy was associated with an increased risk of subsequent composite adverse maternal outcomes in unadjusted (OR, 2.82; 95\% CI, $1.24-6.44 ; p=0.013)$ and adjusted models (OR, 2.64; $95 \%$ CI, 1.07-6.47; $p=0.034$ ) (Table 3).

In subgroup analysis, patients with a history of adverse pregnancy had higher risk of subsequent adverse maternal outcomes in those with serum albumin $<3.5 \mathrm{~g} / \mathrm{dL}$ (OR, 5.06; 95\% CI, 1.43-17.83; $p=0.012$ ), proteinuria great than $1 \mathrm{~g} /$ day (OR, 6.02; 95\% CI, 1.15-31.63; $p=0.034$ ), and diagnosis of hypertension at kidney biopsy (OR, 2.83; 95\% CI, 1.11-7.19; $p=0.029$ ). There was no relationship between history of adverse pregnancy and subsequent adverse maternal outcome in patients with different kidney functions at the time of kidney biopsy (Fig. 1).

\section{Kidney Function and Proteinuria after Delivery}

There were 19 patients with 21 pregnancies without follow-up data. During a median (25th, 75th) follow-up of 2.2 


\begin{tabular}{|c|c|c|c|c|c|}
\hline Subgroup & No. of pregnancy & No. of Event & OR & & $p$-value \\
\hline \multicolumn{6}{|l|}{ Serum albumin levels } \\
\hline Serum albumin $<3.5 \mathrm{~g} / \mathrm{dL}$ & 59 & 37 & 5.06 & $\longrightarrow$ & 0.012 \\
\hline Serum albumin $\geq 3.5 \mathrm{~g} / \mathrm{dL}$ & 41 & 22 & 2.73 & $\rightarrow$ & 0.15 \\
\hline \multicolumn{6}{|l|}{$24 \mathrm{~h}$ urine protein excretion } \\
\hline Proteinuria $\geq 1 \mathrm{~g} /$ day & 35 & 15 & 6.02 & $\longrightarrow$ & 0.034 \\
\hline Proteinuria $<1 \mathrm{~g} /$ day & 65 & 44 & 2.39 & - & 0.15 \\
\hline \multicolumn{6}{|l|}{ estimated GFR levels } \\
\hline $\mathrm{eGFR}<60 \mathrm{~mL} / \mathrm{min} / 1.73 \mathrm{~m}^{2}$ & 10 & 5 & 16.00 & $\longrightarrow$ & 0.08 \\
\hline $\mathrm{eGFR} \geq 60 \mathrm{~mL} / \mathrm{min} / 1.73 \mathrm{~m}^{2}$ & 90 & 54 & 1.89 & $=$ & 0.18 \\
\hline \multicolumn{6}{|l|}{ History of hypertension } \\
\hline With hypertension & 21 & 16 & 2.83 & $\rightarrow$ & 0.029 \\
\hline Without hypertension & 79 & 43 & 3.44 & $\rightarrow$ & 0.41 \\
\hline & & & & 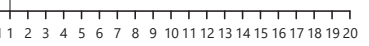 & \\
\hline
\end{tabular}

Fig. 1. Subgroup analysis of a history of adverse pregnancy with fetal and maternal outcomes. GFR, glomerular filtration rate; eGFR, estimated glomerular filtration rate.

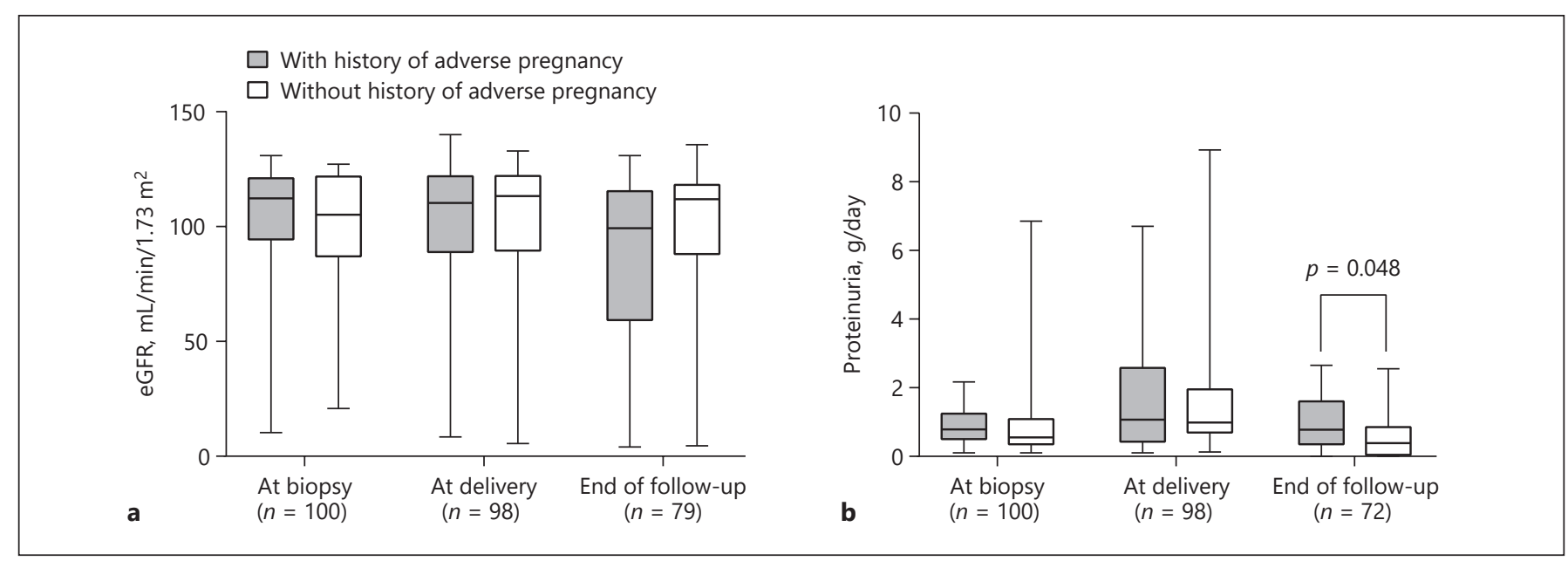

Fig. 2. Changes in eGFR (a) and protein urine (b) at the time of kidney biopsy, during delivery and after delivery. eGFR, estimated glomerular filtration rate.

(1.1-4.7) years after delivery, the eGFR levels were not different between the 2 groups $(99.3[59.3,115.4] \mathrm{mL} / \mathrm{min} /$ $1.73 \mathrm{~m}^{2}$ vs. $112.0[88.0,118.2] \mathrm{mL} / \mathrm{min} / 1.73 \mathrm{~m}^{2}, p=0.06$ ) (Fig. 2a; online suppl. Table 2); while the 24 -h urine protein excretion in patients with a history of adverse pregnancy was borderline higher than those without a history of adverse pregnancy $(0.78[0.36,1.61] \mathrm{g} /$ day vs. $0.39[0.05,0.85]$ g/day, $p=0.048)$ (Fig. 2b; online suppl. Table 2). 


\section{Discussion}

In the current study, we demonstrated that a history of adverse pregnancy in pregnant patients with IgAN was not associated with subsequent adverse fetal outcomes, but associated with an increased risk of subsequent adverse maternal outcomes, especially in those with potential risk factors, including decreased serum albumin, overt proteinuria, and hypertension. At the end of followup, the levels of eGFR were not different between the 2 groups, while the proteinuria levels were borderline higher in patients with a history of adverse pregnancy than those without a history of adverse pregnancy.

Several studies have evaluated the possible relationship between obstetrical complications and adverse pregnancy outcomes in later pregnancies. A nationwide Swedish cohort including 354,676 women without predisposing chronic diseases showed that women with previous preterm preeclampsia had a higher risk of adverse pregnancy outcomes, including stillbirth, placental abruption, and preterm births in a second pregnancy [12]. Recently, a large population-based registry study reported similar results, that women who deliver with obstetrical complications (including pre-eclampsia, placental abruption, stillbirth, neonatal death, and small for gestational age infants) in a first birth even at term had substantially increased risks of subsequent preterm delivery [14]. These studies indicated the persistent causal factors in the mother and the possibility of shared biologic linking pregnancy conditions and outcomes $[12,14]$, due to the disorders of placental function (such as ischemic placental disease) $[14,15]$. Evidence also showed that pregnancy-related complications were independent risk factors for kidney and other cardio or cerebrovascular disease outcomes $[9,16]$. It indicated that metabolic, endothelial, and inflammatory changes may be responsible for the early onset of subsequent disease propensity in patients who experience a complicated pregnancy [16]. IgAN is an autoimmune renal disease arising from consequences of increased levels of galactose-deficient circulating IgA1. The mechanisms involved the formation of pathogenic IgA1-containing immune complexes in intercapillary and mesangial, which causes the cell activation and initiation of glomerular injury [17]. Therefore, IgAN patients with pregnancy may have such impact of immunologic microenvironment on maternal-fetal interface. However, whether the presence of history of adverse pregnancy in pregnant patients with IgAN could increase the risk of unfavorable maternal-fetal interface has not been elaborated in previous studies.

Pregnancy in IgA Nephropathy
In this study, we separately defined the history of adverse pregnancy and subsequent maternal and fetal outcomes as a composition instead of a single exposure, which was different from previous studies $[12,14,18]$. Our study demonstrated that a history of adverse pregnancy in IgAN patients was associated with an increased risk of subsequent adverse maternal outcomes, but not for adverse fetal outcomes. The potential interpretation was that women with a history of adverse pregnancy had more extensive follow-up during subsequent pregnancy compared to those without such history. One recent meta-analysis showed IgAN had high risk of preeclampsia (OR, 11.80; 95\% CI, 7.53-18.48), pregnancy-induced hypertension (OR, 10.39; 95\% CI, 5.45-19.80), and preterm birth (OR, 3.37; 95\% CI, 1.91-5.95) and low birth weight (OR, 2.36; 95\% CI, 1.52-3.66) [19]. However, the increased risk of adverse fetal outcomes was not correspondingly high as that of preeclampsia and pregnancyinduced hypertension [19]. Similar results were reported by Piccoli et al. [20] that there was a higher risk of preeclampsia in IgAN pregnancy patients, but the risk of preterm delivery was not increased. Such a discrepancy indicated that the occurrence of "late" or "maternal" preeclampsia in IgAN pregnancy patients had less effect on early fetal growth. The occurrence of adverse maternal outcomes in the present study was similar as "late" or "maternal" preeclampsia, which may result in less severely fetal growth or shorten gestation.

Our study also noted that a history of adverse pregnancy was associated with increased risks of adverse maternal outcomes in those with a decreased serum albumin, overt proteinuria, and pre-existing hypertension, although the sample size of subgroup analysis was small. The possible explanation was that adverse maternal outcomes and renal progression shared common risk factors. Previous studies have found that proteinuria during pregnancy, hypertensive disorders, and decreased kidney function were key risk factors associated with adverse maternal outcomes [21-23]. Although we cannot prove a causal relationship in this study, the presence of history of adverse pregnancy was associated with subsequent adverse maternal outcomes after adjustment for multiple risk factors in IgAN patients. Interventions to reduce proteinuria, optimal blood pressure control, and balance nutrition for these high-risk mothers were required during the course of pregnancy.

There were several limitations in the present study. First, this retrospective cohort study was conducted in a single Chinese center. We could not exclude the possibility of residual confounding from unmeasured or un- 
known covariates and selection bias because of the nature of retrospective analysis with limited sample size. In addition, the demonstration of the effect of the present study was limited to Chinese pregnancy IgAN patients. Therefore, it should be interpreted with caution for patients with other kidney diseases and races. Second, few patients with $\mathrm{eGFR}<60 \mathrm{~mL} / \mathrm{min} / 1.73 \mathrm{~m}^{2}$ were involved, which limited the study's power to assess adverse pregnancy outcomes in those with decreased level of eGFR. Third, we only have the information of RAS blocker and immunosuppressive agents at the time of kidney biopsy, but had no corresponding information during the course of pregnancy and follow-up, which may place restrictions on research of treatment strategy. However, due to limited evidence regarding on the effect of the history of adverse pregnancy on recurrent maternal-outcomes in IgAN patients, it is important to highlight the requirement for further studies on the topic.

In conclusion, a history of adverse pregnancy in pregnant patients with IgAN was associated with a high risk for adverse maternal outcomes in subsequent pregnancy, while it was not associated with increased risk of adverse fetal outcomes. After delivery, both eGFR and proteinuria levels were stable at the end of follow-up. Further studies with large sample size and long-term follow-up are needed.

\section{Statement of Ethics}

This study was approved by the First Affiliated Hospital of Sun Yat-sen University Institutional Review Boards, Guangzhou, China (No. [2020] 207). All participants provided their written informed consent prior to inclusion in the registry.

\section{Conflict of Interest Statement}

The results presented in this article have not been published previously except in abstract form. The authors declare no relevant conflicts of interest. Co-author Xueqing $\mathrm{Yu}$ is an Editorial Board Member of Kidney Diseases.

\section{Funding Sources}

This work was supported by Grants from the Chinese National Key Technology R and D Program, Ministry of Science and Technology (No. 2017YFC0907601, No. 2017YFC0907602, and No. 2017YFC0907603, No. 2016YFC0906100, No. 2016YFC0906101 to W.C.); the National Natural Science Foundation of China (No. 81970599 to W.C.); Guangzhou Municipal Program of Science and Technology (No. 201804020049 to W.C.); Key Laboratory of National Health Commission, and Key Laboratory of Nephrology, Guangdong Province, Guangzhou, China (No. 2002B60118, 2017B030314019 to W.C.).

\section{Author Contributions}

W.C. and X.Q.Y. were involved in the research idea and study design; X.J.L. and L.F. analyzed data and interpreted and drafted the manuscript; X.N., C.W., and Y.L. contributed to data acquisition; W.F.C. was involved in supervision or mentorship. All authors were involved in the revision of manuscript and responsible for providing intellectual content of critical importance to the work described as well as for final approval of the version to be published.

\section{Data Availability Statement}

All data generated or analyzed during this study are included in this article and its online supplementary files. Further inquiries can be directed to the corresponding author.

\section{References}

1 Li J, Cui Z, Long J, Huang W, Wang J, Zhang $\mathrm{H}$, et al. Primary glomerular nephropathy among hospitalized patients in a national database in China. Nephrol Dial Transplant. 2018;33(12):2173-81.

2 Le W, Liang S, Hu Y, Deng K, Bao H, Zeng $\mathrm{C}$, et al. Long-term renal survival and related risk factors in patients with IgA nephropathy: results from a cohort of 1155 cases in a Chinese adult population. Nephrol Dial Transplant. 2012;27(4): 1479-85.

3 Floege J, Amann K. Primary glomerulonephritides. Lancet. 2016;387:2036-48.

4 O'Shaughnessy MM, Hogan SL, Poulton CJ, Falk RJ, Singh HK, Nickeleit V, et al. Temporal and demographic trends in glomerular disease epidemiology in the Southeastern
United States, 1986-2015. Clin J Am Soc Nephrol. 2017;12:614-23.

5 Lykke JA, Paidas MJ, Langhoff-Roos J. Recurring complications in second pregnancy. Obstet Gynecol. 2009;113:1217-24.

6 Malacova E, Regan A, Nassar N, RaynesGreenow C, Leonard H, Srinivasjois R, et al. Risk of stillbirth, preterm delivery, and fetal growth restriction following exposure in a previous birth: systematic review and metaanalysis. BJOG. 2018;125:183-92.

7 Wang F, Lu JD, Zhu Y, Wang TT, Xue J. Renal outcomes of pregnant patients with immunoglobulin A nephropathy: a systematic review and meta-analysis. Am J Nephrol. 2019;49: 214-24.

8 Su X, Lv J, Liu Y, Wang J, Ma X, Shi S, et al. Pregnancy and kidney outcomes in patients with IgA nephropathy: a cohort study. Am J Kidney Dis. 2017;70:262-9.

9 Park S, Yoo KD, Park JS, Hong JS, Baek S, Park SK, et al. Pregnancy in women with immunoglobulin A nephropathy: are obstetrical complications associated with renal prognosis? Nephrol Dial Transplant. 2018;33:45965.

10 Levey AS, Stevens LA, Schmid CH, Zhang YL, Castro AF 3rd, Feldman HI, et al. A new equation to estimate glomerular filtration rate. Ann Intern Med. 2009;150:604-12.

11 Trimarchi H, Barratt J, Cattran DC, Cook HT, Coppo R, Haas M, et al. Oxford classification of IgA nephropathy 2016: an update from the IgA Nephropathy Classification Working Group. Kidney Int. 2017;91(5): 1014-21. 
12 Wikstrm AK, Stephansson O, Cnattingius S. Previous preeclampsia and risks of adverse outcomes in subsequent nonpreeclamptic pregnancies. Am J Obstet Gynecol. 2011;204: 148.e1-6.

13 Blencowe H, Cousens S, Oestergaard MZ Chou D, Moller A-B, Narwal R, et al. National, regional, and worldwide estimates of preterm birth rates in the year 2010 with time trends since 1990 for selected countries: a systematic analysis and implications. Lancet. 2012;379:2162-72.

14 Kvalvik LG, Wilcox AJ, Skjærven R, Østbye T, Harmon QE. Term complications and subsequent risk of preterm birth: registry based study. BMJ. 2020;369:m1007.

15 Ananth CV, Peltier MR, Chavez MR, Kirby RS, Getahun D, Vintzileos AM. Recurrence of ischemic placental disease. Obstet Gynecol. 2007;110:128-33.
16 Jasper R, Skelding K. Cardiovascular disease risk unmasked by pregnancy complications. Eur J Intern Med. 2018;57:1-6.

17 Suzuki H, Kiryluk K, Novak J, Moldoveanu Z, Herr AB, Renfrow MB, et al. The pathophysiology of IgA nephropathy. J Am Soc Nephrol. 2011;22:1795-803.

18 Ahrens KA, Rossen LM, Branum AM. Pregnancy loss history at first parity and selected adverse pregnancy outcomes. Ann Epidemiol. 2016;26:474-81.e9.

19 Piccoli G, Kooij I, Attini R, Montersino B, Fassio F, Gerbino M, et al. A systematic review on materno-foetal outcomes in pregnant women with IgA nephropathy: a case of "latematernal” preeclampsia? J Clin Med. 2018;7: 212.
20 Piccoli GB, Attini R, Cabiddu G, Kooij I, Fassio F, Gerbino M, et al. Maternal-foetal outcomes in pregnant women with glomerulonephritides. Are all glomerulonephritides alike in pregnancy? J Autoimmun. 2017;79:91-8.

21 Kendrick J, Sharma S, Holmen J, Palit S, Nuccio E, Chonchol M. Kidney disease and maternal and fetal outcomes in pregnancy. Am J kidney Dis. 2015;66(1):55-9.

22 Morris RK, Riley RD, Doug M, Deeks JJ, Kilby MD. Diagnostic accuracy of spot urinary protein and albumin to creatinine ratios for detection of significant proteinuria or adverse pregnancy outcome in patients with suspected pre-eclampsia: systematic review and meta-analysis. BMJ. 2012;345:e4342.

23 Ukah UV, De Silva DA, Payne B, Magee LA, Hutcheon JA, Brown H, et al. Prediction of adverse maternal outcomes from pre-eclampsia and other hypertensive disorders of pregnancy: a systematic review. Pregnancy Hypertens. 2018;11:115-23. 